patients resistant or intolerant to imatinib. Results from a real lifebased Italian multicenter retrospective study on 114 patients. Am J Hematol. 2010;85(12):960-963.

15. Clark RE, Polydoros F, Apperley JF, et al. De-escalation of tyrosine kinase inhibitor dose in patients with chronic myeloid leukaemia with stable major molecular response (DESTINY): an interim analysis of a non-randomised, phase 2 trial. Lancet Haematol. 2017;4(7):e310-e316.

16. Naqvi K, Jabbour E, Skinner J, et al. Early results of lower dose dasa- tinib (50 mg daily) as frontline therapy for newly diagnosed chronicphase chronic myeloid leukemia. Cancer. 2018;124(13):2740-2747.

17. Schiffer CA. The evolution of dasatinib dosage over the years and its relevance to other anticancer medications. Cancer. 2018;124(13): 2687-2689.

18. Fassoni AC, Baldow C, Roeder I, Glauche I. Reduced tyrosine kinase inhibitor dose is predicted to be as effective as standard dose in chronic myeloid leukemia: a simulation study based on phase III trial data. Haematologica. 2018;103(11):1825-1834.

\title{
The only thing that stops a bad microbiome, is a good microbiome
}

\section{Jessica R. Galloway-Peñ $a^{1,2}$ and Robert R. Jen $q^{1,3}$}

${ }^{1}$ Department of Genomic Medicine; ${ }^{2}$ Department of Infectious Diseases, Infection Control and Employee Health and ${ }^{3}$ Department of Stem Cell Transplantation, The University of Texas MD Anderson Cancer Center, Houston, TX, USA.

E-mail:jrgalloway@mdanderson.org/rrjenq@mdanderson.org

doi:10.3324/haematol.2019.222430

M ultidrug resistant (MDR) bacterial colonization in the gut is frequently induced by excessive use of antibiotics. ${ }^{1}$ Fecal microbiota transplantation (FMT) has been shown to be quite successful in treating refractory and recurrent Clostridium difficile infection. ${ }^{2}$ Thus, current research is focusing on how FMT may also help in decolonizing MDR organisms (MDRO) and in preventing recurrent $\mathrm{MDR}$ infections. ${ }^{3}$ Decolonization of MDRO via FMT may be particularly useful in patients with hematologic malignancies, such as those undergoing hematopoietic stem cell transplantation (HSCT), ${ }^{4}$ as use of chemotherapeutic agents and frequent administration of antibiotics can favor the selection of resistant pathogens. ${ }^{5,6}$

In spite of the increasing evidence that the feasibility and safety of FMT in immunocompromised cohorts is comparable to that of immunocompetent patients, administering FMT in the setting of hematologic malignancy remains a cause for concern due to perceived risks of translocation and sepsis. ${ }^{7,8}$ Given the growing body of literature associating a dysbiotic microbiome with adverse HSCT outcomes and treatment-related toxicities, including infection, delivering a diverse microbiome via FMT to immunocompromised patients may provide a variety of benefits, such as promoting colonization resistance and reducing the risk of bacterial translocation. Thus, attempts to better characterize the safety and efficacy of FMT in these patients are merited.

In this issue of the Journal, Battipaglia et al. ${ }^{10}$ describe a retrospective case series of 10 patients with hematologic malignancies undergoing FMT for MDRO colonization before or after allogeneic HSCT. In this study, the authors show both the safety and efficacy of using FMT for decolonization of carbapenem-resistant Enterobactericeae (CRE), carbapenem-resistant Pseudomonas (CRP), and vancomycin-resistant Enterococcus (VRE). Notably, the study reports FMT both pre- and post transplant. The majority of patients who received FMT prior to transplant did not have recurrent MDRO even after HSCT, indicating the prophylactic use of FMT. Interestingly, the procedure remained effective for long- term MDRO decolonization in the majority of patients despite the use of broad-spectrum antibiotics in some of the patients after FMT. This implies that FMT can potentially achieve full decolonization of MDRO rather than merely reducing the levels of MDRO below the limit of detection.

While FMT was shown to be successful in decolonizing the MDRO studied, the FMT did not always prevent additional post-transplant infections from other bacteria susceptible to antibiotics. Interestingly, only $50 \%$ of patients concomitantly colonized with extended spectrum $\beta$-lactamase (ESBL)-producing Enterobacteriaceae obtained decolonization. This is reminiscent of a case report by Stalenhoef et al. where FMT successfully eradicated a Pseudomonas aeruginosa urinary tract infection, while stool cultures remained positive for extended-spectrum $\beta$-lactamase (ESBL)-producing Enterobacteriaceae three months after FMT. ${ }^{11}$ Thus, the higher frequency of failure of FMT to eradicate the ESBL-producing Enterobacteriaceae in these two studies may suggest potential limitations to this therapy.

Although the specific mechanisms underlying the success of FMT for MDRO colonization remain unclear, Figure 1 depicts an overview of the general concepts regarding the use of FMT for MDRO in patients with hematologic malignancies. Given that this study looked specifically at CRE, CRP, and VRE, it remains unclear if other MDRO may be equally responsive to FMT. Furthermore, given the seemingly discrepant results for CRE, CRP, and VRE compared to ESBL-producing organisms, one might consider that distinct mechanisms of action underlie how FMT mediates response for different MDRO.

Due to the retrospective nature of the study, in contrast to a controlled study, it is unclear how physicians decided to treat each patient with FMT case by case. Moreover, there was a large variation between cases in the time of FMT relative to HSCT and the MDRO colonization/infection in both pre- and post-HSCT groups. Thus, it remains to be determined what the ideal timeframe for FMT is in both scenarios. The use of relat- 


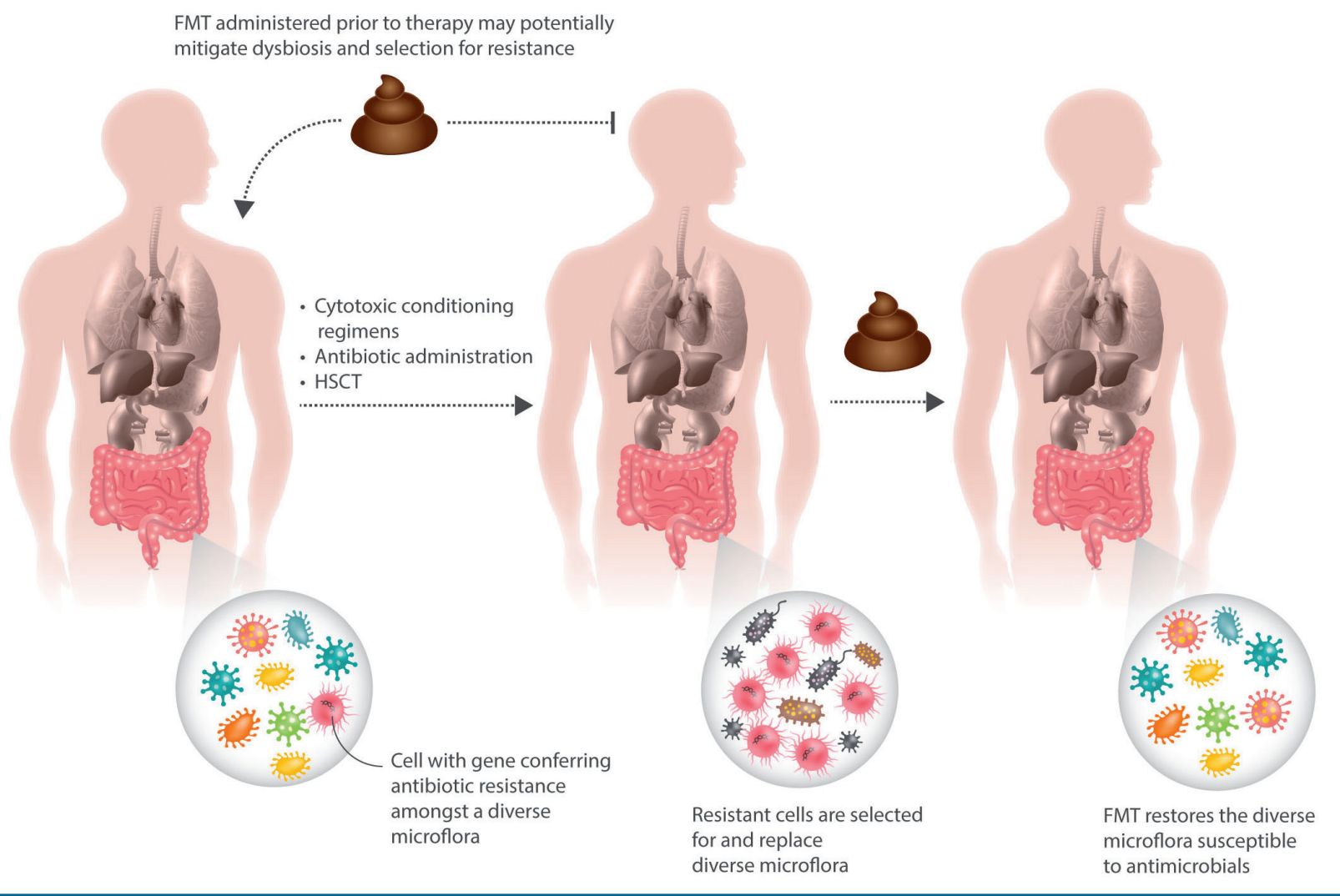

Figure 1. Fecal microbiota transplant (FMT) as a potential decolonization strategy for multi-drug resistant organisms (MDRO) in hematologic malignancy patients. After cytotoxic chemotherapy, hematopoietic stem cell transplantation (HSCT), and antibiotic administration, the likelihood that resistant bacteria replace diverse microbiota increases. FMT can potentially restore susceptibility to antimicrobials by replacing resistant bacteria with a diverse microflora when used as a decolonization strategy in response to infection or colonization with MDRO. When administered prior to cancer treatment, FMT may potentially mitigate dysbiosis and selection of resistance.

ed donors was preferred as it was perceived that common environmental exposures would reduce additional risk of transferring infectious agents between the donor and recipient. Intriguingly, neither of the two cases using an unrelated donor was successfully decolonized; given so few numbers, we cannot determine if this result is significant. Consequently, the best choice of donor remains to be explored. If it were to be found that related donors are in fact preferable to universal donors, appropriate screening and regulations will be an important consideration in the future.

One critical piece missing from this study is the understanding of the microbiome in this process. Although theoretically FMT decolonization works via restoration of microbial diversity leading to colonization resistance and displacement of the MDRO, ${ }^{12,13}$ experimentally showing what micro-organisms were important for decolonization in each case, which organisms presented robust and durable colonization, as well as if resistance genes were completely displaced after FMT would strengthen these types of studies and vastly improve the understanding of the mechanism by which FMT decolonizes MDRO. This represents an important future opportunity for investigators.

With MDR infections set to be the world's leading cause of morbidity and mortality by the year 2050 , set to surpass even cancer, and with few new antimicrobials in the pipeline, the need for novel and different approaches to treat MDR infections is critical. ${ }^{14}$ Moreover, we need to improve our clinical understanding of the antibiotic resistome, particularly in immunocompromised patients who experience repeated exposures to antimicrobials. Although no large randomized controlled trials have been performed to study the efficacy and safety of FMT for MDR organisms in the immunocompromised patient, this study and others have provided some promising evidence, and suggest that a non-antibiotic therapy for MDR colonization and infections may become common practice in the future for patients with hematologic malignancies. ${ }^{15-17}$ The use of FMT as a decolonization agent both as a prophylactic and treatment measure may prove effective in preventing MDR outbreaks and transmission, prolonged in-hospital care, recurrent infection, and improve the overall outcomes of HSCT patients. Given the numerous potential benefits, and demonstrated safety and efficacy, the fear and negative perception of FMT in the cancer setting is unjustified. ${ }^{15}$

\section{Acknowledgments}

JGP is supported by the NIH (K01 AI143881). RRJ is supported by the NIH (R01 HL124112) and the Cancer Prevention and Research Institute of Texas (RR160089). 


\section{References}

1. Modi SR, Collins JJ, Relman DA. Antibiotics and the gut microbiota J Clin Invest. 2014; 124(10):4212-4218.

2. Quraishi MN, Widlak M, Bhala N et al. Systematic review with meta-analysis: the efficacy of faecal microbiota transplantation for the treatment of recurrent and refractory Clostridium difficile infection. Aliment Pharmacol Ther. 2017;46(5):479-493.

3. Saha S, Tariq R, Tosh PK, et al. Faecal microbiota transplantation for eradicating carriage of multidrug-resistant organisms: a systematic review. Clin Microbiol Infect. 2019;25(8):958-963.

4. Bilinski J, Grzesiowski P, Sorensen N, et al. Fecal microbiota transplantation in patients with blood disorders inhibits gut colonization with antibiotic-resistant bacteria: results of a prospective, single-center study. Clin Infect Dis. 2017;65(3):364-370.

5. Galloway-Pena J, Brumlow C, Shelburne S. Impact of the microbiota on bacterial infections during cancer treatment. Trends Microbiol. 2017;25(12):992-1004

6. Montassier E, Gastinne T, Vangay P, et al. Chemotherapy-driven dysbiosis in the intestinal microbiome. Aliment Pharmacol Ther. 2015;42(5):515-528.

7. Shogbesan O, Poudel DR, Victor S, et al. A systematic review of the efficacy and safety of fecal microbiota transplant for Clostridium difficile infection in immunocompromised patients. Can J Gastroenterol Hepatol. 2018;2018:1394379.

8. Wang S, Xu M, Wang W, et al. Systematic review: adverse events of fecal microbiota transplantation. PLoS One. 2016;11(8):e0161174.

9. Shono Y, van den Brink MRM. Gut microbiota injury in allogeneic haematopoietic stem cell transplantation. Nat Rev Cancer.
2018;18(5):283-295.

10. Battipaglia G, Malard F, Rubio MT, et al. Fecal microbiota transplantation before or after allogeneic hematopoietic transplantation in patients with hematologic malignancies carrying multidrug-resistance bacteria. Haematologica 2019;104(8):1682-1688.

11. Stalenhoef JE, Terveer EM, Knetsch CW, et al. Fecal microbiota transfer for multidrug-resistant gram-negatives: a clinical success combined with microbiological failure. Open Forum Infect Dis 2017;4(2): ofx047.

12. Kelly CR, Kahn S, Kashyap P, et al. Update on fecal microbiota transplantation 2015: indications, methodologies, mechanisms, and outlook. Gastroenterology. 2015;149(1):223-237.

13. Khoruts A, Sadowsky MJ. Understanding the mechanisms of faecal microbiota transplantation. Nat Rev Gastroenterol Hepatol. 2016;13(9):508-516.

14. O'Neill J. Review on antimicrobial resistance antimicrobial resistance: tackling a crisis for the health and wealth of nations. Review on Antimicrobial Resistance. London, 2014. Available from: http://amr-review.org

15. Abu-Sbeih H, Ali FS, Wang Y. Clinical review on the utility of fecal microbiota transplantation in immunocompromised patients. Curr Gastroenterol Rep. 2019;21(4):8

16. Wardill HR, Secombe KR, Bryant RV, et al. Adjunctive fecal microbiota transplantation in supportive oncology: Emerging indications and considerations in immunocompromised patients. EBioMedicine. 2019;44 730-740.

17. DeFilipp Z, Hohmann E, Jenq RR, et al. Fecal microbiota transplantation: restoring the injured microbiome after allogeneic hematopoietic cell transplantation. Biol Blood Marrow Transplant. 2019;25(1) e17-e22.

\section{Predicting risk for recurrence of arterial ischemic stroke in children: thrombophilia as another piece of the puzzle}

\section{Ghada Aborkhees and Lesley Gayle Mitchell}

Department of Pediatrics, University of Alberta, Edmonton, AB, Canada

E-mail: LESLEY GAYLE MITCHELL - Lesley.Mitchell@albertahealthservices.ca

doi:10.3324/haematol.2019.222695

$\mathrm{R}$ ecurrent arterial ischemic stroke (AIS) is increasingly recognized as a significant cause of mortality and morbidity in the pediatric population. Identifying risk factors for recurrent AIS is essential for developing strategies for secondary stroke prevention. While multiple risk factors have been identified for AIS events, the only confirmed risk factor for initial AIS recurrence is the presence of vasculopathy, particularly moyamoya disease. ${ }^{1-3}$ In a meta-analysis, prothrombotic risk factors were found to be associated with AIS in pediatric patients. ${ }^{4}$ However, the role of thrombophilia as an independent risk factor for recurrent AIS has not been established due to a paucity of research in the area and the lack of statistical power in the published studies.

In the current edition of Haematologica, deVeber et al. report on an international prospective cohort study which recruited 894 pediatric patients from centers in Germany, Canada and the UK. ${ }^{5}$ The primary objective of the study was to determine the association of prothrombotic risk factors and/or underlying stroke subtypes with risk for recurrent stroke. The authors excluded asymptomatic strokes and transient ischemic attacks due to the difference in underlying disease as well as the differing outcomes from symptomatic strokes. Sickle cell disease and moyamoya vasculopathies were also excluded as their recurrence rates and risk factors differ from those of other subtypes of pediatric AIS. The authors report an overall AIS recurrence rate of $17.9 \%$ in the cohort studied. The study confirmed the association of vasculopathy as a risk factor for AIS recurrence. The novel approach in the current study was the examination of the role of thrombophilia as an independent risk factor for AIS recurrence. Study patients were excluded if they had thrombophilic markers with established pathophysiological relevance such as homozygous protein $\mathrm{C}$ and homozygous antithrombin deficiency. Analysis of the study data showed that the following were independent risk factors for recurrence: antithrombin deficiency (hazard ratio 3.9; $95 \%$ confidence interval: 1.4-10.9), increased lipoprotein(a) (hazard ratio 2.3; 95\% confidence interval: 1.3-4.1) and more than one prothrombotic marker (hazard ratio 1.9; $95 \%$ confidence interval: $1.1-3.2)$. The results obtained from this study highlight the importance of screening AIS cases for thrombophilia in order to identify the children at risk of AIS recurrence.

The reported study is a valuable addition to the previous efforts to identify the risk factors for AIS recurrence. There are significant strengths of the study design. The first was the relatively large sample size, which provided adequate power to determine the association of pro- 